\title{
WAVE INDUCED OSCILLATORY AND STEADY FLOWS \\ IN THE ANNULUS OF A CATHETERIZED VISCOELASTIC TUBE
}

\author{
MA Ye, NG Chiu-On ${ }^{1}, \quad$ CHANG Yin-Yee \\ Department of Mechanical Engineering, \\ The University of Hong Kong, Pokfulam Road, Hong Kong, China
}

April 1, 2010

\begin{abstract}
A perturbation analysis based on equations of motion in Lagrangian form is presented for the oscillatory and time-mean viscous flows induced by a propagating wave of small amplitude in an annulus with a viscoelastic outer wall. Owing to the steady streaming effect, the existence of a catheter in a blood vessel brings in an additional steady pressure gradient, a correction to that predicted by the linear theory, and an additional steady shear stress, which may increase the possibility of hemolysis of red blood cells. The steady pressure gradient is found as an analytical function of the Lagrangian radial coordinate, which is in sharp contrast to the Eulerian description by which the steady pressure gradient is constant across the section. The effects of catheter size and oscillation frequency on the wave speed and wave attenuation in the first order, and on the time-mean steady quantities like velocity, pressure gradient, and shear stress in the second order are studied. Results of the present study suggest that a catheter radius that is some $30 \%$ of the outer tube radius is a possibly critical value, above which the mean pressure gradient and the mean shear stresses induced in the fluid could be too large to render the catheter unsafe to use.
\end{abstract}

Keywords: annulus flow; steady streaming; catheterized artery

\footnotetext{
${ }^{1}$ Corresponding author. Fax: +852 2858 5415; E-mail: cong@hku.hk.
} 


\section{Introduction}

The study of oscillatory flow of a viscous fluid contained in a flexible tube is of importance particularly to biomechanics. In clinical situations such as balloon angioplasty, a pressure transducer with a long fine catheter is inserted in a peripheral artery to measure the pressure gradient over a large part of the arterial tree during angioplasty procedures $^{[1]}$. The insertion of catheters in blood vessels will, however, alter the pressure distribution, thereby inducing errors to the pressure gradient measurement ${ }^{[2]}$. Also, hemolysis of blood samples is a common clinical problem encountered in the Emergency Department (ED). Excessively large shear stress in the annulus flow due to catheterization may destroy the red blood cells, leading to inaccuracy in assay results and often to the need for repeated blood draws ${ }^{[3]}$.

With an objective to understand blood flow in an annular region through a stenotic artery, several studies have been performed to estimate the correction to the measured values of blood pressure gradient ${ }^{[1,2,4,5,6]}$. By the nonlinear phenomenon known as steady streaming, which amounts to a time-independent component of flow ${ }^{[7]}$, there exists a higher-order non-zero time-mean pressure gradient in addition to the oscillatory component found at the leading order. Sarkar and Jayaraman ${ }^{[1]}$ derived a correction to the mean pressure drop as predicted by the linear theory in pulsatile flow through a catheterized stenosed rigid artery. The effects of the catheter size and oscillation frequency on the time-mean quantities such as velocity, impedance and wall shear stress were studied. A nonlinear analysis of the annulus flow in an elastic tube was further carried out by Sarkar and Jayaraman ${ }^{[2]}$. They also showed that the mean pressure gradient would change with catheter size at all frequencies. The geometry as well as the elasticity of the wall could play an important role in the dynamics of the flow even for small catheter radius. More complex effects, such as artery clot, artery curvature and non-Newtonian fluid, were investigated by Jayaraman and Sarkar ${ }^{[4]}$, Jayaraman and Dash ${ }^{[5]}$ and Sankar ${ }^{[6]}$, respectively. The associated mass transport problem in oscillatory flow through a catheterized artery was also studied by, e.g., Sarkar and Jayaraman ${ }^{[8]}$.

The above-mentioned works are based on the Eulerian formulation, in which the 
Taylor expansion theorem is employed to approximate the boundary conditions on a moving wall by those on the mean position of the wall ${ }^{[9]}$. Such an approach would, however, limit the validity of the second-order results to waves of extremely small amplitude when the oscillation frequency is high ${ }^{[10]}$. To circumvent this problem, we adopt in this work the Lagrangian description, by which the conditions on a moving boundary are prescribed exactly by referring to its undisturbed position ${ }^{[11]}$. The Lagrangian description is intrinsically particle specific, and hence its analysis will yield results that can be interpreted as the conditions experienced by individual particles, e.g., blood cells, in the flow. The Lagrangian steady streaming velocity, which show the time-mean movement of particles, is found directly by this approach. Ma and $\mathrm{Ng}^{[10]}$ recently developed a Lagrangian model to investigate the nonlinear flow induced by oscillatory pressure forcing through a thick-walled flexible tube without catheter insertion. Their mathematical formulation is largely followed in this work. In particular, their solutions for oscillatory as well as time-mean motions with zero initial wall stresses will be used as particular cases for comparison in this paper.

Sharp and Mohammad ${ }^{[3]}$ found that the probability of hemolysis would increase as a result of increasing pressure difference and catheter size. They defined a threshold for hemolysis of red blood cells as a function of exposure time and shear stress. Below the threshold, hemolysis is much less probable to occur. In this regard, it is essential to determine a catheter size that will not cause the blood cells to be subject to excessively large shear stress during the process of catheterization. This has motivated the present study.

Specifically, the present work is to study the oscillatory and time-mean flows, as induced by purely oscillatory pressure forcing, in a catheterized artery with viscoelastic outer wall. The Lagrangian coordinates are employed. The catheter, or the inner tube, is assumed to be rigid, and the condition outside the outer tube can be either stress-free (i.e., a free tube without constraint by the surrounding tissues), or zerodisplacement axially (i.e., a tethered tube constrained by the surrounding tissues). Effects of non-Newtonian fluid, stenosis, curvature, gravity and initial stresses of the outer wall are not considered in this work. A perturbation analysis is performed by introducing a small ratio of wave amplitude to tube radius. The wavenumber and 
wave attenuation are found in the first order and the steady fluid dynamic parameters like the axial mass transport velocity, pressure gradient, and shear stress are found in the second order. Results are generated to illustrate the effects of oscillation frequency and catheter radius on the first-order oscillatory flow and the second-order steady quantities. Finally, based on the results, a critical catheter radius is suggested.

\section{Mathematical formulation}

Figure 1 shows a schematic diagram of the annular geometry and the cylindrical coordinate system. The radius of the rigid catheter, and the initial inner and outer radii of the outer wall are represented by $d, a$ and $b$, respectively. By the Lagrangian description, the instantaneous radial and axial coordinates of a particle of fixed identity, $(r, z)$, and the pressure, $p$, are functions of the initial coordinates of the particle, $(R, Z)$, and time, $t$. Axisymmetry is assumed, and hence any dependence on the azimuthal position $\theta$ is eliminated. As a result of an oscillatory pressure gradient of angular frequency $\sigma$, a progressive wave of wavenumber $k$ is induced in the system, where $k$ and $\sigma$ are related by a dispersion equation, as will be deduced later.

A linear Voigt viscoelastic model is proposed for the tube wall: the Cauchy stress $\mathbf{T}$ is a linear combination of viscous and elastic parts ${ }^{[12,11,13]}$ :

$$
\mathbf{T}=-p \mathbf{I}+2 \mu \mathbf{D}+G \mathbf{E}
$$

where $\mathbf{I}$ is the identity tensor, $\mu$ is the classical Newtonian viscosity, $\mathbf{D}=\frac{1}{2}\left(\mathbf{L}+\mathbf{L}^{\mathrm{T}}\right)$ is the rate of deformation tensor, $\mathbf{L}=\dot{\mathbf{F}} \cdot \mathbf{F}^{-1}$ is the velocity gradient tensor, $G$ is the shear modulus, and $\mathbf{E}=\mathbf{B}-\mathbf{I}$ is the strain tensor, in which $\mathbf{F}$ is the deformation gradient tensor of material, and $\mathbf{B}=\mathbf{F} \cdot \mathbf{F}^{\mathrm{T}}$ is the Finger or left Cauchy-Green tensor $^{[13,14]}$. In its limit when $\mu=0$, the constitutive relation (1) represents a neoHookean elastic solid. In a sense, the neo-Hookean law generalizes Hooke's law. While the latter involves only linear strains and is good for infinitesimally small deformations, the former contains quadratic strains and may describe fairly large deformations of an isotropic material. Some previous works, e.g., Demiray ${ }^{[15]}$ and 
Gundiah $^{[16]}$, have clearly remarked that the neo-Hookean law could be applied in the constitutive relation to model the biological tissues.

We suppose that the tube inner radius, catheter radius and the outer wall thickness are comparable with one another, all of them being much smaller than the wavelength, which is of the order of the tube length $L$. Based on this assumption of sharp contrast in length scales, the following parameter

$$
\epsilon \equiv a / L \ll 1
$$

will be used as an ordering parameter for the perturbation analysis below.

Following $\mathrm{Ma}$ and $\mathrm{Ng}^{[10]}$, we introduce the following normalized variables, which are distinguished by a caret:

$$
\left.\begin{array}{r}
\left(r_{l}, R, b, d\right)=a\left(\hat{r}_{l}, \hat{R}, \hat{b}, \hat{d}\right), \quad\left(z_{l}, Z\right)=L\left(\hat{z}_{l}, \hat{Z}\right), \quad k=L^{-1} \hat{k}, \quad t=\sigma^{-1} \hat{t}, \\
p_{f}=\left(\rho_{f} \sigma^{2} L^{2}\right) \hat{p}_{f}, \quad p_{w}=G_{w} \hat{p}_{w}, \quad N_{f}=\epsilon^{-2} \mu_{f} \sigma \hat{N}_{f}, \quad N_{w}=G_{w} \hat{N}_{w}, \\
\left(\tau_{f r z}, T_{f}\right)=\epsilon^{-1} \mu_{f} \sigma\left(\hat{\tau}_{f r z}, \hat{T}_{f}\right), \quad\left(\tau_{f r r}, \tau_{f \theta \theta}, \tau_{f z z}\right)=\mu_{f} \sigma\left(\hat{\tau}_{f r r}, \hat{\tau}_{f \theta \theta}, \hat{\tau}_{f z z}\right), \\
\left(\tau_{w r z}, T_{w}\right)=\epsilon G_{w}\left(\hat{\tau}_{w r z}, \hat{T}_{w}\right), \quad\left(\tau_{w r r}, \tau_{w \theta \theta}, \tau_{w z z}\right)=G_{w}\left(\hat{\tau}_{w r r}, \hat{\tau}_{w \theta \theta}, \hat{\tau}_{w z z}\right),
\end{array}\right\}
$$

where the subindex $l$ is used to distinguish between the fluid and outer wall domains

$$
l=\left\{\begin{array}{ll}
f & \text { for fluid in } a>R>d \\
w & \text { for wall in } b>R>a
\end{array},\right.
$$

$\rho$ represents the density, $\tau_{i j}$ are the deviatoric stress components, $T$ and $N$ are, respectively, the tangential and normal stress components on a material surface as seen in an $(R, Z)$ plane.

The motions of the fluid and the wall are governed by the continuity and momentum equations in the Lagrangian system as follows. The continuity equation is

$$
\frac{\partial\left(\hat{r}_{l}, \hat{z}_{l}\right)}{\partial(\hat{R}, \hat{Z})}=\frac{\hat{R}}{\hat{r}_{l}}
$$

The momentum equations are

$$
\begin{gathered}
\epsilon^{2} \frac{\partial^{2} \hat{r}_{f}}{\partial \hat{t}^{2}}=-\frac{\hat{r}_{f}}{\hat{R}} \frac{\partial\left(\hat{p}_{f}, \hat{z}_{f}\right)}{\partial(\hat{R}, \hat{Z})}+\frac{\epsilon^{2}}{\alpha^{2}}\left[\frac{1}{\hat{R}} \frac{\partial\left(\hat{r}_{f} \hat{\tau}_{f r r}, \hat{z}_{f}\right)}{\partial(\hat{R}, \hat{Z})}+\frac{\hat{r}_{f}}{\hat{R}} \frac{\partial\left(\hat{r}_{f}, \hat{\tau}_{f r z}\right)}{\partial(\hat{R}, \hat{Z})}-\frac{\hat{\tau}_{f \theta \theta}}{\hat{r}_{f}}\right], \\
\frac{\partial^{2} \hat{z}_{f}}{\partial \hat{t}^{2}}=-\frac{\hat{r}_{f}}{\hat{R}} \frac{\partial\left(\hat{r}_{f}, \hat{p}_{f}\right)}{\partial(\hat{R}, \hat{Z})}+\frac{1}{\alpha^{2}}\left[\frac{1}{\hat{R}} \frac{\partial\left(\hat{r}_{f} \hat{\tau}_{f z r}, \hat{z}_{f}\right)}{\partial(\hat{R}, \hat{Z})}+\epsilon^{2} \frac{\hat{r}_{f}}{\hat{R}} \frac{\partial\left(\hat{r}_{f}, \hat{\tau}_{f z z}\right)}{\partial(\hat{R}, \hat{Z})}\right],
\end{gathered}
$$




$$
\begin{gathered}
\epsilon^{2} \frac{\partial^{2} \hat{r}_{w}}{\partial \hat{t}^{2}}=-\frac{\beta}{\alpha^{4}} \frac{\hat{r}_{w}}{\hat{R}} \frac{\partial\left(\hat{p}_{w}, \hat{z}_{w}\right)}{\partial(\hat{R}, \hat{Z})}+\frac{\beta}{\alpha^{4}}\left[\frac{1}{\hat{R}} \frac{\partial\left(\hat{r}_{w} \hat{\tau}_{w r r}, \hat{z}_{w}\right)}{\partial(\hat{R}, \hat{Z})}+\epsilon^{2} \frac{\hat{r}_{w}}{\hat{R}} \frac{\partial\left(\hat{r}_{w}, \hat{\tau}_{w r z}\right)}{\partial(\hat{R}, \hat{Z})}-\frac{\hat{\tau}_{w \theta \theta}}{\hat{r}_{w}}\right] \\
\frac{\partial^{2} \hat{z}_{w}}{\partial \hat{t}^{2}}=-\frac{\beta}{\alpha^{4}} \frac{\hat{r}_{w}}{\hat{R}} \frac{\partial\left(\hat{r}_{w}, \hat{p}_{w}\right)}{\partial(\hat{R}, \hat{Z})}+\frac{\beta}{\alpha^{4}}\left[\frac{1}{\hat{R}} \frac{\partial\left(\hat{r}_{w} \hat{\tau}_{w z r}, \hat{z}_{w}\right)}{\partial(\hat{R}, \hat{Z})}+\frac{\hat{r}_{w}}{\hat{R}} \frac{\partial\left(\hat{r}_{w}, \hat{\tau}_{w z z}\right)}{\partial(\hat{R}, \hat{Z})}\right]
\end{gathered}
$$

The dimensionless stress components are given as below:

$$
\begin{gathered}
\hat{\tau}_{f r r}=2 \frac{\hat{r}_{f}}{\hat{R}} \frac{\partial\left(\hat{\dot{r}}_{f}, \hat{z}_{f}\right)}{\partial(\hat{R}, \hat{Z})}, \quad \hat{\tau}_{f \theta \theta}=2 \frac{\hat{\dot{r}}_{f}}{\hat{r}_{f}}, \quad \hat{\tau}_{f z z}=2 \frac{\hat{r}_{f}}{\hat{R}} \frac{\partial\left(\hat{r}_{f}, \hat{\dot{z}}_{f}\right)}{\partial(\hat{R}, \hat{Z})}, \\
\hat{\tau}_{f r z}=\hat{\tau}_{f z r}=\frac{\hat{r}_{f}}{\hat{R}}\left[\epsilon^{2} \frac{\partial\left(\hat{r}_{f}, \hat{\dot{r}}_{f}\right)}{\partial(\hat{R}, \hat{Z})}+\frac{\partial\left(\hat{\dot{z}}_{f}, \hat{z}_{f}\right)}{\partial(\hat{R}, \hat{Z})}\right], \\
\hat{\tau}_{w r r}=2 \frac{\alpha^{2} \gamma}{\beta} \frac{\hat{r}_{w}}{\hat{R}} \frac{\partial\left(\hat{\dot{r}}_{w}, \hat{z}_{w}\right)}{\partial(\hat{R}, \hat{Z})}+\left[\left(\frac{\partial \hat{r}_{w}}{\partial \hat{R}}\right)^{2}+\epsilon^{2}\left(\frac{\partial \hat{r}_{w}}{\partial \hat{Z}}\right)^{2}-1\right], \\
\hat{\tau}_{w z z}=2 \frac{\alpha^{2} \gamma}{\beta} \frac{\hat{r}_{w}}{\hat{R}} \frac{\partial\left(\hat{r}_{w}, \hat{\dot{z}}_{w}\right)}{\partial(\hat{R}, \hat{Z})}+\left[\epsilon^{2} \gamma\left(\frac{\hat{\dot{r}}_{w}}{\hat{r}_{w}}+\left[\left(\frac{\partial \hat{r}_{w}}{\partial \hat{R}}\right)^{2}-1\right],\left(\frac{\partial \hat{z}_{w}}{\partial \hat{Z}}\right)^{2}-1\right],\right.
\end{gathered}
$$

and

$$
\begin{aligned}
\hat{\tau}_{w r z}=\hat{\tau}_{w z r}= & \frac{\alpha^{2} \gamma}{\beta} \frac{\hat{r}_{w}}{\hat{R}}\left[\frac{\partial\left(\hat{r}_{w}, \hat{\dot{r}}_{w}\right)}{\partial(\hat{R}, \hat{Z})}+\epsilon^{-2} \frac{\partial\left(\hat{\dot{z}}_{w}, \hat{z}_{w}\right)}{\partial(\hat{R}, \hat{Z})}\right] \\
& +\left[\epsilon^{-2}\left(\frac{\partial \hat{r}_{w}}{\partial \hat{R}}\right)\left(\frac{\partial \hat{z}_{w}}{\partial \hat{R}}\right)+\left(\frac{\partial \hat{r}_{w}}{\partial \hat{Z}}\right)\left(\frac{\partial \hat{z}_{w}}{\partial \hat{Z}}\right)\right] .
\end{aligned}
$$

More details about the deduction of the Lagrangian equations of motions in the cylindrical system can be found in $\mathrm{Ma}$ and $\mathrm{Ng}^{[10]}$. In the equations above, three dimensionless parameters are introduced:

$$
\alpha=a\left(\frac{\sigma}{\nu_{f}}\right)^{\frac{1}{2}}, \quad \beta=\frac{\epsilon^{2} a^{2} G_{w}}{\rho_{w} \nu_{f}^{2}}, \quad \gamma=\frac{\epsilon^{2} \nu_{w}}{\nu_{f}} .
$$

where $\nu_{l}=\mu_{l} / \rho_{l}$ is the kinematic viscosity. These parameters have the following physical meanings: $\alpha$ is a frequency parameter known as the Womersley number; $\beta$ represents the significance of the tube elasticity; $\gamma$ is a ratio of the tube viscosity to the fluid viscosity.

The normalized boundary conditions read as follows:

$$
\begin{gathered}
\hat{r}_{f}=0, \quad \hat{z}_{f}=0, \quad \text { on } \hat{R}=\hat{d} \\
\hat{r}_{f}=\hat{r}_{w}, \quad \hat{z}_{f}=\hat{z}_{w}, \quad \frac{\alpha^{2}}{M \beta} \hat{T}_{f}=\hat{T}_{w}, \quad \frac{\alpha^{2}}{M \beta} \hat{N}_{f}=\hat{N}_{w}, \quad \text { on } \hat{R}=1,
\end{gathered}
$$




$$
\hat{T}_{w}=0, \quad \hat{N}_{w}=0, \quad \text { on } \hat{R}=\hat{b}
$$

where

$$
M=\frac{\rho_{w}}{\rho_{f}}=O(1),
$$

which represents the ratio of wall density to fluid density. We remark that the no-slip condition (17) becomes invalid when $\hat{d}=0$, for which the no-stress condition should be applied instead. Reference should be made to $\mathrm{Ma}$ and $\mathrm{Ng}^{[10]}$ for the case without the inner tube.

The dimensionless expressions for $\hat{T}_{l}$ and $\hat{N}_{l}$ are

$$
\begin{aligned}
\hat{T}_{f}|n|^{2}= & \epsilon^{2}\left(\hat{\tau}_{f r r}-\hat{\tau}_{f z z}\right) \frac{\partial \hat{r}_{f}}{\partial \hat{Z}} \frac{\partial \hat{z}_{f}}{\partial \hat{Z}}+\hat{\tau}_{f z r}\left[\left(\frac{\partial \hat{z}_{f}}{\partial \hat{Z}}\right)^{2}-\epsilon^{2}\left(\frac{\partial \hat{r}_{f}}{\partial \hat{Z}}\right)^{2}\right], \\
\hat{N}_{f}|n|^{2}= & \left(-\alpha^{2} \hat{p}_{f}+\epsilon^{2} \hat{\tau}_{f r r}\right)\left(\frac{\partial \hat{z}_{f}}{\partial \hat{Z}}\right)^{2}+\epsilon^{2}\left(-\alpha^{2} \hat{p}_{f}+\epsilon^{2} \hat{\tau}_{f z z}\right)\left(\frac{\partial \hat{r}_{f}}{\partial \hat{Z}}\right)^{2} \\
& -2 \epsilon^{2} \hat{\tau}_{f r z} \frac{\partial \hat{r}_{f}}{\partial \hat{Z}} \frac{\partial \hat{z}_{f}}{\partial \hat{Z}}, \\
\hat{T}_{w}|n|^{2}= & \left(\hat{\tau}_{w r r}-\hat{\tau}_{w z z}\right) \frac{\partial \hat{r}_{w}}{\partial \hat{Z}} \frac{\partial \hat{z}_{w}}{\partial \hat{Z}}+\hat{\tau}_{w z r}\left[\left(\frac{\partial \hat{z}_{w}}{\partial \hat{Z}}\right)^{2}-\epsilon^{2}\left(\frac{\partial \hat{r}_{w}}{\partial \hat{Z}}\right)^{2}\right],
\end{aligned}
$$

and

$$
\begin{aligned}
\hat{N}_{w}|n|^{2}= & \left(-\hat{p}_{w}+\hat{\tau}_{w r r}\right)\left(\frac{\partial \hat{z}_{w}}{\partial \hat{Z}}\right)^{2}+\epsilon^{2}\left(-\hat{p}_{w}+\hat{\tau}_{w z z}\right)\left(\frac{\partial \hat{r}_{w}}{\partial \hat{Z}}\right)^{2} \\
& -2 \epsilon^{2} \hat{\tau}_{w r z} \frac{\partial \hat{r}_{w}}{\partial \hat{Z}} \frac{\partial \hat{z}_{w}}{\partial \hat{Z}} .
\end{aligned}
$$

where $|n|=\left[(\partial r / \partial Z)^{2}+(\partial z / \partial Z)^{2}\right]^{1 / 2}$ is the length of the unit tangential vector of a material curve $\mathbf{S}=(r(R, Z, t), z(R, Z, t))$.

On assuming that the induced radial and axial displacements in both the fluid and the wall are one order of magnitude smaller than the corresponding scalings, we may follow Pierson ${ }^{[17]}$ in expanding the variables into powers of $\epsilon$ :

$$
\begin{aligned}
\left(\hat{r}_{l}, \hat{z}_{l}\right)=(\hat{R}, \hat{Z})+ & \epsilon\left(\hat{r}_{l 1}, \hat{z}_{l 1}\right)+\epsilon^{2}\left(\hat{r}_{l 2}, \hat{z}_{l 2}\right)+\cdots, \\
\hat{p}_{l}= & \epsilon \hat{p}_{l 1}+\epsilon^{2} \hat{p}_{l 2}+\cdots, \\
\left(\hat{\tau}_{l r r}, \hat{\tau}_{l \theta \theta}, \hat{\tau}_{l z z}, \hat{\tau}_{l r z}\right)= & \epsilon\left(\hat{\tau}_{l r r 1}, \hat{\tau}_{l \theta \theta 1}, \hat{\tau}_{l z z 1}, \hat{\tau}_{l r z 1}\right) \\
& +\epsilon^{2}\left(\hat{\tau}_{l r r 2}, \hat{\tau}_{l \theta \theta 2}, \hat{\tau}_{l z z 2}, \hat{\tau}_{l r z 2}\right)+\cdots,
\end{aligned}
$$




$$
\begin{aligned}
& \hat{z}_{w 1}(\hat{R}, \hat{Z}, \hat{t})=\hat{z}_{w 11}(\hat{Z}, \hat{t})+\epsilon^{2} \hat{z}_{w 12}(\hat{R}, \hat{Z}, \hat{t}), \\
& \hat{z}_{w 2}(\hat{R}, \hat{Z}, \hat{t})=\hat{z}_{w 21}(\hat{Z}, \hat{t})+\epsilon^{2} \hat{z}_{w 22}(\hat{R}, \hat{Z}, \hat{t})
\end{aligned}
$$

where the leading terms $\hat{z}_{w 11}$ and $\hat{z}_{w 21}$ are independent of $\hat{R}$. The $O(\epsilon)$ terms are the leading-order displacements consisting of purely first harmonic components, while the higher-order terms consist of higher harmonics as well as steady components.

Substitution of the expansions above into the governing equations (5)-(9), the stress components (10)-(15) and the boundary conditions (17)-(19), and collecting terms of equal power of $\epsilon$, we may obtain the first- and second-order problems as detailed in the following sections.

\section{First-order problem}

At $O(\epsilon)$, the governing equations for the fluid are:

$$
\begin{gathered}
\frac{\partial \hat{z}_{f 1}}{\partial \hat{Z}}+\frac{\partial \hat{r}_{f 1}}{\partial \hat{R}}+\frac{\hat{r}_{f 1}}{\hat{R}}=0 \\
\frac{\partial \hat{p}_{f 1}}{\partial \hat{R}}=0 \\
\frac{\partial^{2} \hat{z}_{f 1}}{\partial \hat{t}^{2}}=-\frac{\partial \hat{p}_{f 1}}{\partial \hat{Z}}+\frac{1}{\alpha^{2}}\left[\frac{\partial \hat{\tau}_{f r z 1}}{\partial \hat{R}}+\frac{\hat{\tau}_{f r z 1}}{\hat{R}}\right],
\end{gathered}
$$

and those for the tube wall are:

$$
\begin{gathered}
\frac{\partial \hat{z}_{w 11}}{\partial \hat{Z}}+\frac{\partial \hat{r}_{w 1}}{\partial \hat{R}}+\frac{\hat{r}_{w 1}}{\hat{R}}=0, \\
-\frac{\partial \hat{p}_{w 1}}{\partial \hat{R}}+\frac{\partial \hat{\tau}_{w r r 1}}{\partial \hat{R}}+\frac{\hat{\tau}_{w r r 1}-\hat{\tau}_{w \theta \theta 1}}{\hat{R}}=0, \\
\frac{\alpha^{4}}{\beta} \frac{\partial^{2} \hat{z}_{w 11}}{\partial \hat{t}^{2}}=-\frac{\partial \hat{p}_{w 1}}{\partial \hat{Z}}+\frac{\partial \hat{\tau}_{w r z 1}}{\partial \hat{R}}+\frac{\hat{\tau}_{w r z 1}}{\hat{R}}+\frac{\partial \hat{\tau}_{w z z 1}}{\partial \hat{Z}},
\end{gathered}
$$

where the stress components $\hat{\tau}_{l i j 1}$ are given as follows:

$$
\begin{array}{rlrl}
\hat{\tau}_{f r r 1} & =2 \frac{\partial \hat{\dot{r}}_{f 1}}{\partial \hat{R}}, & \hat{\tau}_{f \theta \theta 1} & =2 \frac{\hat{\dot{r}}_{f 1}}{\hat{R}}, \\
\hat{\tau}_{f z z 1}=2 \frac{\partial \hat{\dot{z}}_{f 1}}{\partial \hat{Z}}, & \hat{\tau}_{f r z 1}=\frac{\partial \hat{\dot{z}}_{f 1}}{\partial \hat{R}},
\end{array}
$$




$$
\begin{gathered}
\hat{\tau}_{w r r 1}=2 \frac{\alpha^{2} \gamma}{\beta} \frac{\partial \hat{\dot{r}}_{w 1}}{\partial \hat{R}}+2 \frac{\partial \hat{r}_{w 1}}{\partial \hat{R}}, \\
\hat{\tau}_{w \theta \theta 1}=2 \frac{\alpha^{2} \gamma}{\beta} \frac{\hat{\dot{r}}_{w 1}}{\hat{R}}+2 \frac{\hat{r}_{w 1}}{\hat{R}}, \\
\hat{\tau}_{w z z 1}=2 \frac{\alpha^{2} \gamma}{\beta} \frac{\partial \hat{\dot{\xi}}_{w 11}}{\partial \hat{Z}}+2 \frac{\partial \hat{z}_{w 11}}{\partial \hat{Z}},
\end{gathered}
$$

and

$$
\hat{\tau}_{w r z 1}=\frac{\alpha^{2} \gamma}{\beta}\left(\frac{\partial \hat{\dot{r}}_{w 1}}{\partial \hat{Z}}+\frac{\partial \hat{\dot{z}}_{w 12}}{\partial \hat{R}}\right)+\frac{\partial \hat{r}_{w 1}}{\partial \hat{Z}}+\frac{\partial \hat{z}_{w 12}}{\partial \hat{R}}
$$

The first-order boundary conditions are

$$
\left.\begin{array}{c}
\hat{r}_{f 1}=0, \hat{z}_{f 1}=0 \quad \text { on } \hat{R}=\hat{d}, \\
\hat{r}_{f 1}=\hat{r}_{w 1}, \quad \hat{z}_{f 1}=\hat{z}_{w 11}, \\
\frac{\alpha^{2}}{M \beta} \hat{\tau}_{f r z 1}=\hat{\tau}_{w r z 1}, \\
-\frac{\alpha^{4}}{M \beta} \hat{p}_{f 1}=-\hat{p}_{w 1}+\hat{\tau}_{w r r 1}
\end{array}\right\} \text { on } \hat{R}=1,
$$

and

$$
\left.\begin{array}{cc}
\text { Case 1: } & \hat{\tau}_{w r z 1}=0, \\
\text { Case 2: } & \hat{z}_{w 11}=0, \\
-\hat{p}_{w 1}+\hat{\tau}_{w r r 1}=0
\end{array}\right\} \text { on } \hat{R}=\hat{b}
$$

In the outer boundary condition above, case 1 represents a free tube, where the stresses on the outer wall are absent, while in case 2 the tube is tethered by the perivascular tissues so that the longitudinal motion of the outer surface is strongly constrained and the leading order of the axial displacement can be taken as zero ${ }^{[18]}$.

We suppose that the pressure forcing that is applied to the fluid at the origin $\hat{p}_{f 1}(\hat{Z}=0)$ is a simple harmonic function of $\hat{t}$, and as a result, a traveling wave is induced in the fluid:

$$
\hat{p}_{f 1}=\operatorname{Re}\left[p^{o} \mathrm{e}^{\mathrm{i}(\hat{t}-\hat{k} \hat{Z})}\right]
$$

where Re stands for the real part, $p^{\circ}$ is a known constant equal to the amplitude of the pressure forcing at $\hat{Z}=0$, and $\mathrm{i}=\sqrt{-1}$ is the complex unit. To seek the solution, let us now express the first-order quantities in the same harmonic form as $\hat{p}_{f 1}$ :

$$
\left(\hat{r}_{f 1}, \hat{z}_{f 1}, \hat{r}_{w 1}, \hat{z}_{w 11}, \hat{z}_{w 12}, \hat{p}_{w 1}\right)=\operatorname{Re}\left[\left(r_{f}^{o}, z_{f}^{o}, r_{w}^{o}, z_{w c}^{o}, z_{w v}^{o}, p_{w}^{o}\right) \mathrm{e}^{\mathrm{i}(\hat{t}-\hat{k} \hat{Z})}\right]
$$


where $r_{f}^{o}, z_{f}^{o}, r_{w}^{o}, z_{w v}^{o}$ and $p_{w}^{o}$ are complex functions of $\hat{R}$, and $z_{w c}^{o}$ is an unknown constant to be determined by the boundary conditions. On substituting these expressions into the governing equations, the following general solutions satisfying Eq. (42) can be deduced:

$$
\begin{gathered}
z_{f}^{o}(\hat{R})=-\mathrm{i} \hat{k} p^{o}+\lambda J_{0}(\lambda \hat{R}) B_{1}+\lambda Y_{0}(\lambda \hat{R}) B_{6}, \\
r_{f}^{o}(\hat{R})=\frac{\hat{k}^{2} \hat{R}}{2} p^{o}+\mathrm{i} \hat{k} J_{1}(\lambda \hat{R}) B_{1}+\mathrm{i} \hat{k} Y_{1}(\lambda \hat{R}) B_{6}+\frac{B_{7}}{\hat{R}}, \\
z_{w c}^{o}=B_{4}, \\
r_{w}^{o}(\hat{R})=\frac{\mathrm{i} \hat{k} \hat{R}}{2} B_{4}+\frac{B_{2}}{\hat{R}}, \\
p_{w}^{o}=\frac{\zeta}{\beta} \mathrm{i} \hat{k} B_{5}, \\
z_{w v}^{o}(\hat{R})=\left[\hat{k}^{2}\left(B_{4}+B_{5}\right)-\frac{\alpha}{\zeta} B_{4}\right] \frac{\hat{R}^{2}}{4}+\ln (\hat{R}) B_{3},
\end{gathered}
$$

where $\lambda=\alpha \mathrm{i}^{3 / 2}, \zeta=\alpha^{2} \gamma \mathrm{i}+\beta, J_{n}$ and $Y_{n}$ are respectively the Bessel functions of the first and second kinds of order $n$, and the complex integration constants $B_{1}-B_{7}$ as well as the complex wavenumber $\hat{k}$ are to be determined by the boundary conditions.

In case 1 for a free tube, substituting the general solutions into the boundary conditions (43)-(44) results in a homogeneous linear algebraic system of equations for the eight constants $p^{o}$ and $B_{1}-B_{7}$ :

$$
A X=0
$$

where the matrix $A$ and vector $X$ are given as below:

$$
A=\left(\begin{array}{cccc}
-\mathrm{i} \hat{k} & \lambda J_{0}(\lambda \hat{d}) & 0 & 0 \\
\hat{k}^{2} \hat{d}^{2} / 2 & \mathrm{i} \hat{k} \hat{d} J_{1}(\lambda \hat{d}) & 0 & 0 \\
\hat{k}^{2} / 2 & \mathrm{i} \hat{k} J_{1}(\lambda) & -1 & 0 \\
-\mathrm{i} \hat{k} & \lambda J_{0}(\lambda) & 0 & 0 \\
0 & \alpha^{4} J_{1}(\lambda) /(M \zeta) & -\mathrm{i} \hat{k} & 1 \\
\alpha^{4} /(M \zeta) & 0 & -2 & 0 \\
0 & 0 & -\mathrm{i} \hat{k} & 1 \\
0 & 0 & -2 / \hat{b}^{2} & 0
\end{array}\right.
$$




$$
\begin{aligned}
& \left.\begin{array}{cccc}
0 & 0 & \lambda Y_{0}(\lambda \hat{d}) & 0 \\
0 & 0 & \mathrm{i} \hat{k} \hat{d} Y_{1}(\lambda \hat{d}) & 1 \\
-1 & 0 & \lambda Y_{0}(\lambda) & 0 \\
-\mathrm{i} \hat{k} / 2 & 0 & \mathrm{i} \hat{k} Y_{1}(\lambda) & 1 \\
\hat{k}^{2}-\alpha^{4} /(2 \zeta) & \hat{k}^{2} / 2 & \alpha^{4} Y_{1}(\lambda) /(M \zeta) & 0 \\
\mathrm{i} \hat{k} & -\mathrm{i} \hat{k} & 0 & 0 \\
\hat{k}^{2} \hat{b}^{2}-\alpha^{4} \hat{b}^{2} /(2 \zeta) & \hat{k}^{2} \hat{b}^{2} / 2 & 0 & 0 \\
\mathrm{i} \hat{k} & -\mathrm{i} \hat{k} & 0 & 0
\end{array}\right), \\
& X=\left(\begin{array}{llllllll}
p^{o} & B_{1} & B_{2} & B_{3} & B_{4} & B_{5} & B_{6} & B_{7}
\end{array}\right)^{T} .
\end{aligned}
$$

The system will have a non-trivial solution if and only if the determinant of the coefficient matrix $A$ vanishes:

$$
|A|=0
$$

This condition yields the characteristic equation governing the eigenvalue, which is the complex wavenumber $\hat{k}$. Equation (56) admits four complex solutions for $\hat{k}=\hat{k}_{r}+\mathrm{i} \hat{k}_{i}$, where the real part $\hat{k}_{r}$ denotes the wavenumber, and the imaginary part $\hat{k}_{i}$ is the attenuation constant. Our focus is on the forward traveling waves, so we only consider the two solutions with $\hat{k}_{r}>0$ and $\hat{k}_{i}<0$. Each of these two solutions corresponds to a distinct wave mode. The wave with a slower phase velocity $C_{1}=\sigma / k_{r 1}$ is called Young wave representing a pressure wave propagating in the fluid, while the one with a higher phase velocity $C_{2}=\sigma / k_{r 2}$ is called Lamb wave representing a wave traveling largely along the wall. Most of our discussions below will be on the Young wave mode, which is more important in the present study.

The constants $B_{1}-B_{7}$ are related to the pressure amplitude $p^{o}$ by the following relations:

$$
\frac{p^{o}}{\triangle_{11}}=\frac{B_{1}}{\triangle_{12}}=\frac{B_{2}}{\triangle_{13}}=\frac{B_{3}}{\triangle_{14}}=\frac{B_{4}}{\triangle_{15}}=\frac{B_{5}}{\triangle_{16}}=\frac{B_{6}}{\triangle_{17}}=\frac{B_{7}}{\triangle_{18}}
$$

where $\triangle_{k l}$ is the cofactor obtained by eliminating the $k$ th row and $l$ th column of $|A|$.

In case 2 for a tethered tube, $B_{4}=0$ and the dispersion equation becomes much 
simpler, which can be expressed as follows:

$$
\hat{k}^{2}=\left[\lambda Y_{0}(\lambda \hat{d})-\frac{\lambda J_{0}(\lambda \hat{d}) Y_{0}(\lambda)}{J_{0}(\lambda)}\right] \frac{\hat{b}^{2} \alpha^{4}}{2 M \zeta\left(\hat{b}^{2}-1\right)\left(K_{1}-K_{2}\right)},
$$

where

$$
\begin{aligned}
K_{1}= & {\left[1-\frac{J_{0}(\lambda \hat{d})}{J_{0}(\lambda)}\right]\left\{\hat{d} Y_{1}(\lambda \hat{d})-\frac{\hat{d} J_{1}(\lambda \hat{d}) Y_{0}(\lambda)}{J_{0}(\lambda)}\right.} \\
& \left.-\left[\frac{\lambda J_{0}(\lambda)}{2}-J_{1}(\lambda)\right] \frac{Y_{0}(\lambda)}{J_{0}(\lambda)}+\frac{\lambda Y_{0}(\lambda)}{2}-Y_{1}(\lambda)\right\},
\end{aligned}
$$

and

$$
K_{2}=\left[\lambda Y_{0}(\lambda \hat{d})-\frac{\lambda J_{0}(\lambda \hat{d}) Y_{0}(\lambda)}{J_{0}(\lambda)}\right]\left[\frac{\hat{d}^{2}-1}{2}-\frac{\hat{d} J_{1}(\lambda \hat{d})}{\lambda J_{0}(\lambda)}+\frac{J_{1}(\lambda)}{\lambda J_{0}(\lambda)}\right]
$$

The Lamb wave does not exist this time and only the Young wave can survive in the tethering case.

Some numerical results are presented in Fig. 2 to demonstrate the effects of the catheter radius $\hat{d}$ on the Young wave speed $C_{1}$, and the wave damping (given by the imaginary part of the wavenumber $\hat{k}_{i}$ ) as functions of the Womersley number $\alpha$. The physical inputs according to the properties of a blood vessel are chosen as ${ }^{[19]}$ : $a=2 \times 10^{-3} \mathrm{~m}, L=2 \times 10^{-1} \mathrm{~m}, \hat{b}=b / a=1.15, \rho_{f}=1.056 \times 10^{3} \mathrm{~kg} / \mathrm{m}^{3}$, $\rho_{w}=1.1 \times 10^{3} \mathrm{~kg} / \mathrm{m}^{3}$ (or $M=1.0417$ ), $\mu_{f}=3.3 \times 10^{-3} \mathrm{Pas}, \mu_{w}=33 \mathrm{Pas}$, and $G_{w}=5.371 \times 10^{5} \mathrm{~Pa}(\beta=20000$ and $\gamma=1)$. As for the size of a catheter, Sarkar and Jayaraman $^{[2]}$ pointed out that the catheter is normally $0.66-2.6 \mathrm{~mm}$ in diameter, while the blood vessel is $3-5.7 \mathrm{~mm}$ in diameter. Sarkar and Jayaraman ${ }^{[1]}$ have also considered the ratio of catheter to vessel size being in the range of $0.2-0.5$. Following these works, we here consider a similar range, $0.1-0.5$, for the ratio of the two diameters, which should represent typical catheterization during angioplasty procedures and blood assays.

Figure 2 shows that the Young wave speed is higher and the wave damping is stronger for a tethered tube than those for a free tube, which is consistent with the results obtained by $\mathrm{Ma}$ and $\mathrm{Ng}^{[10]}$. On the other hand, the Young wave speed will be slowed down and the wave attenuation will be enhanced, by increasing the catheter radius. The results for the limiting case $\hat{d}=0$ (i.e., a full circular cross section instead of an annulus) shown in Fig. 2 are obtained using the model by Ma and $\mathrm{Ng}^{[10]}$. As 
remarked earlier, this limiting case is unavailable in the present model, since in the absence of the catheter the no-slip condition can no longer be applied at the inner boundary.

\section{Second-order problem}

The Voigt viscoelastic model is mechanically represented by a spring and a dashpot operating in parallel. A Voigt material, when subjected to a steady load, will first deform at a rate controlled by the dashpot damping, but ultimately will reach a finite deformation controlled by the spring elasticity. $\mathrm{Ma}$ and $\mathrm{Ng}^{[10]}$ have shown that for a viscoelastic material with sufficient elastic strength such that $\mu_{w} / G_{w} \leq O\left(\sigma^{-1}\right)$, or the creeping time for the transient motion is at most comparable to the wave period, the time-mean motion of the tube is short lived with little practical significance; the ultimate steady deformation can be readily attained within several wave periods or so. Under this condition, we only need to find the second-order steady streaming as influenced by the wall viscoelasticity and catheter radius through the first-order oscillatory flow.

At $\mathrm{O}\left(\epsilon^{2}\right)$, the governing equations for the fluid after time-averaging are

$$
\begin{gathered}
\frac{\partial \overline{\hat{\tilde{z}}}_{l 2}}{\partial \hat{Z}}+\frac{\partial \overline{\hat{\hat{r}}}_{l 2}}{\partial \hat{R}}+\frac{\overline{\hat{\hat{r}}}_{l 2}}{\hat{R}}=0, \\
\frac{\partial \overline{\hat{p}}_{f 2}}{\partial \hat{R}}=\Gamma \mathrm{e}^{2 \hat{k}_{i} \hat{Z}}, \\
\alpha^{2} \frac{\partial \overline{\hat{\hat{p}}}_{f 2}}{\partial \hat{Z}}-\frac{\partial^{2} \overline{\hat{\tilde{z}}}_{f 2}}{\partial \hat{R}^{2}}-\frac{1}{\hat{R}} \frac{\partial \overline{\hat{\tilde{z}}}_{f 2}}{\partial \hat{R}}=H \mathrm{e}^{2 \hat{k}_{i} \hat{Z}},
\end{gathered}
$$

where

$$
\begin{gathered}
\Gamma=\frac{1}{2} \operatorname{Re}\left\{\mathrm{i} \hat{k}^{*} p_{f}^{o *} \frac{\mathrm{d} z_{f}^{o}}{\mathrm{~d} \hat{R}}\right\} \\
H=\frac{1}{2} \operatorname{Re}\left\{\alpha^{2}|\hat{k}|^{2} p_{f}^{o *} z_{f}^{o}-\frac{2 \hat{k}^{*}}{\hat{R}} \frac{\mathrm{d}}{\mathrm{d} \hat{R}}\left(\hat{R} z_{f}^{o *} \frac{\mathrm{d} z_{f}^{o}}{\mathrm{~d} \hat{R}}\right)-\hat{k}\left|\frac{\mathrm{d} z_{f}^{o}}{\mathrm{~d} \hat{R}}\right|^{2}\right. \\
\left.+\mathrm{i}\left(\frac{\mathrm{d} r_{f}^{o *}}{\mathrm{~d} \hat{R}}-\frac{r_{f}^{o *}}{\hat{R}}\right)\left(\frac{1}{\hat{R}} \frac{\mathrm{d} z_{f}^{o}}{\mathrm{~d} \hat{R}}-\frac{\mathrm{d}^{2} z_{f}^{o}}{\mathrm{~d} \hat{R}^{2}}\right)\right\} .
\end{gathered}
$$


The overbar denotes time average over one wave period, and the asterisk denotes the complex conjugate.

According to the forcing terms on the right-hand side of the time-averaged equations, the second-order solutions of the steady motion in both the fluid and the wall can be expressed as follows:

$$
\left(\overline{\hat{\hat{r}}}_{f 2}, \overline{\hat{\tilde{z}}}_{f 2}, \overline{\hat{p}}_{f 2}\right)=\left(U_{R}^{s}, U_{L}^{s}, p_{f}^{s}\right) \mathrm{e}^{2 \hat{k}_{i} \hat{Z}}
$$

where $U_{R}^{s}, U_{L}^{s}$ and $p_{f}^{s}$ are functions of $\hat{R}$ only. The term $\exp \left(2 \hat{k}_{i} \hat{Z}\right)$ indicates that all the second-order steady solutions decay along the axial direction. The strength of the axial decay is represented by the magnitude of imaginary part wavenumber $\hat{k}_{i}$, which is always negative as illustrated in Fig. 2.

The boundary conditions for the steady streaming of the fluid can be written as:

$$
\overline{\hat{\hat{r}}}_{f 2}=0, \quad \overline{\hat{\tilde{z}}}_{f 2}=0, \quad \text { on } \hat{R}=\hat{d}
$$

and

$$
\overline{\hat{\hat{r}}}_{f 2}=0, \quad \overline{\hat{\tilde{z}}}_{f 2}=0, \quad \text { on } \hat{R}=1 \text {. }
$$

Substitution of Eq. (66) into Eqs. (61)-(63) and the boundary conditions (67)(68), the time-mean pressure and axial steady streaming velocity at $\hat{Z}=0$ can be readily obtained as

$$
\begin{gathered}
p_{f}^{s}=\int \Gamma \mathrm{d} \hat{R}+M_{1}, \\
U_{L}^{s}=\int_{1}^{\hat{R}} \frac{1}{\hat{R}} \int_{0}^{\hat{R}}\left(2 \hat{k}_{i} \alpha^{2} p_{f}^{s}-H\right) \hat{R} \mathrm{~d} \hat{R} \mathrm{~d} \hat{R}+M_{2} \ln \hat{R},
\end{gathered}
$$

where the integration constant $M_{1}$ and $M_{2}$ can be determined from the boundary conditions (67) and (68).

Substituting $F, \Gamma$ and $H$ into Eq. (70) results in an expression for $U_{L}^{s}$ as follows:

$$
\begin{aligned}
U_{L}^{s}= & \operatorname{Re}\left\{\lambda p^{o *}\left(\frac{1}{2}|\hat{k}|^{2} i+\hat{k}^{* 2} \mathrm{i}+\hat{k}_{i} \hat{k}^{*}\right)\right. \\
\times & {\left[B_{1}\left(J_{0}(\lambda \hat{R})-J_{0}(\lambda)+J_{1}(\lambda \hat{d}) \lambda \hat{d} \ln \hat{R}\right)\right.} \\
+ & \left.B_{6}\left(Y_{0}(\lambda \hat{R})-Y_{0}(\lambda)+Y_{1}(\lambda \hat{d}) \lambda \hat{d} \ln \hat{R}\right)\right]
\end{aligned}
$$




$$
\begin{aligned}
& -|\lambda|^{2} \lambda \hat{k}^{*}\left[\int_{1}^{\hat{R}}\left(J_{0}^{*}(\lambda \hat{R}) B_{1}^{*}+Y_{0}^{*}(\lambda \hat{R}) B_{6}^{*}\right)\left(J_{1}(\lambda \hat{R}) B_{1}+Y_{1}(\lambda \hat{R}) B_{6}\right) \mathrm{d} \hat{R}\right. \\
& \left.-\hat{d} \ln \hat{R}\left(J_{0}^{*}(\lambda \hat{d}) B_{1}^{*}+Y_{0}^{*}(\lambda \hat{d}) B_{6}^{*}\right)\left(J_{1}(\lambda \hat{d}) B_{1}+Y_{1}(\lambda \hat{d}) B_{6}\right)\right] \\
& -\frac{\hat{k}^{*} \lambda^{2}}{2} \int_{1}^{\hat{R}} \frac{1}{\hat{R}} \int_{\hat{d}}^{\hat{R}}\left[\left(\lambda^{*} J_{0}^{*}(\lambda \hat{R}) \hat{R}-2 J_{1}^{*}(\lambda \hat{R})\right)\left(\lambda Y_{0}(\lambda \hat{R})-\frac{2 Y_{1}(\lambda \hat{R})}{\hat{R}}\right) B_{1}^{*} B_{6}\right. \\
& \left.+\left(\lambda^{*} Y_{0}^{*}(\lambda \hat{R}) \hat{R}-2 Y_{1}^{*}(\lambda \hat{R})\right)\left(\lambda J_{0}(\lambda \hat{R})-\frac{2 J_{1}(\lambda \hat{R})}{\hat{R}}\right) B_{1} B_{6}^{*}\right] \mathrm{d} \hat{R} \mathrm{~d} \hat{R} \\
& +i B_{7}^{*} \lambda^{2} \int_{1}^{\hat{R}} \frac{1}{\hat{R}} \int_{\hat{d}}^{\hat{R}}\left[\left(\frac{\lambda J_{0}(\lambda \hat{R})}{\hat{R}}-\frac{2 J_{1}(\lambda \hat{R})}{\hat{R}^{2}}\right) B_{1}\right. \\
& \left.\left.+\left(\frac{\lambda Y_{0}(\lambda \hat{R})}{\hat{R}}-\frac{2 Y_{1}(\lambda \hat{R})}{\hat{R}^{2}}\right) B_{6}\right] \mathrm{~d} \hat{R} \mathrm{~d} \hat{R}\right\} \\
& +\frac{1}{2} \hat{k}_{r} \alpha^{4} \int_{1}^{\hat{R}} \frac{1}{\hat{R}} \int_{\hat{d}}^{\hat{R}}\left|J_{1}(\lambda \hat{R}) B_{1}+Y_{1}(\lambda \hat{R}) B_{6}\right|^{2} \hat{R} \mathrm{~d} \hat{R} \mathrm{~d} \hat{R} \\
& +\frac{1}{2} \hat{k}_{i} \alpha^{2} \int_{1}^{\hat{R}} \frac{1}{\hat{R}} \int_{\hat{d}}^{\hat{R}}\left[\left|\lambda J_{0}(\lambda \hat{R})-\frac{2 J_{1}(\lambda \hat{R})}{\hat{R}}\right|^{2}\left|B_{1}\right|^{2}\right. \\
& \left.+\left|\lambda Y_{0}(\lambda \hat{R})-\frac{2 Y_{1}(\lambda \hat{R})}{\hat{R}}\right|^{2}\left|B_{6}\right|^{2}\right] \hat{R} \mathrm{~d} \hat{R} \mathrm{~d} \hat{R} \\
& +\frac{1}{8} \hat{k}_{i} \alpha^{2}\left(\hat{R}^{2}-1-2 \hat{d}^{2} \ln \hat{R}\right)\left(4 M_{1}-|\hat{k}|^{2}\left|p^{o}\right|^{2}\right)+M_{2} \ln \hat{R} .
\end{aligned}
$$

The steady Lagrangian drift, which is largely in the axial direction, is now determined. This steady current is affected through the parameter $B_{1}$ by the oscillation frequency and catheter radius, as given in Eq. (57). Our second-order analysis and the resulting expression for the Lagrangian drift in annulus flow appear not to have been reported in the existing literature. By virtue of the Lagrangian approach, the boundary conditions are prescribed exactly on the interfaces, and hence the expressions deduced above are good irrespective of the displacement amplitude of the boundaries.

Numerical results, which are obtained with the computational package Mathcad Version 14, are presented below to help us look into the effects of the catheter radius on the second-order steady quantities. Figure 3 shows the steady axial velocity profiles at $\hat{Z}=0$ for different values of catheter radius $\hat{d}$ and the Womersley number $\alpha$ as given in Eq. (16). The results are based on a typical value of fluid pressure amplitude equal to $20 \mathrm{mmHg}^{[20]}$, which corresponds to $p^{o}=20 \times 133.322 \times\left(\rho_{f} \sigma^{2} L^{2}\right)^{-1} / \epsilon=10343 \alpha^{-4}$, where $p^{o}$ is the first-order pressure amplitude as defined in Eq. (45). In Fig. 3, one may see that the steady streaming velocity will decrease in amplitude as the catheter radius 
increases at a low frequency, say $\alpha=2$. The trend becomes reversed, however, for higher frequencies. Unlike the limiting case without a catheter, the steady streaming velocity in annulus flow has a profile varying strongly across the tube section. This is because the no-slip boundary condition on the catheter retards the nearby fluid motion, thereby steepening the velocity gradient near the catheter. As illustrated in Fig. 3, although the amplitude of steady flow in the tethered-tube case is larger than that in the free-tube case, the steady streaming velocity profiles of these two cases are qualitatively the same. Hence, only the free-tube case is considered for further discussion below.

The cross-sectional distribution of steady pressure gradient $\partial \overline{\hat{p}}_{f 2} / \partial \hat{Z}$ at $\hat{Z}=0$ is shown in Fig. 4 as a function of the catheter radius at various frequencies. Unlike the Eulerian mean pressure gradient, which is uniform across the tube section ${ }^{[2,4]}$, the steady pressure gradient in Lagrangian form is a function of the radial coordinate and exhibits a sharp decrease in magnitude in the middle of the annular region, e.g., $\hat{d}=0.3-0.5$ as shown in Fig. 4. In their analysis on the mean pressure gradient of the annulus flow in a flexible tube, Sarkar and Jayaraman ${ }^{[2]}$ found that the insertion of a catheter would increase the magnitude of steady pressure gradient at any frequency. Our results as shown in Fig. 4 are in qualitative agreement with their findings. The quantitative values of our results are of course different from theirs, owing to a different system of description being used. The Lagrangian values shown here are of practical significance, as they can directly reflect the conditions experienced by individual particles.

Figure 5 shows the shear stress distribution in the fluid: $\overline{\hat{\tau}}_{f r z 2}=\tau_{f}^{s} \exp \left(2 \hat{k}_{i} \hat{Z}\right)$ at $\hat{Z}=0$, where



and the steady wall shear stress on the interface between fluid and the inner surface of the outer wall $\tau_{w s s}=\left.\tau_{f}^{s}\right|_{\hat{R}=1}$, and shear stress on the catheter surface $\tau_{c s s}=\left.\tau_{f}^{s}\right|_{\hat{R}=\hat{d}}$ due to the steady streaming. The presence of a catheter is to dramatically change the distribution of the shear stress in the annular region, as clearly shown in Fig. 5. In particular, the shear stress near the bounding walls is appreciably enhanced as the catheter size increases. Again, such similar effects of catheter size on the wall 
shear stress have been reported previously by Sarkar and Jayaraman ${ }^{[2]}$. We here confirm that catheterization can indeed lead to intensification of the steady shear stress, especially near the walls. This needs to be taken into consideration when one is concerned with, e.g., hemolysis of red blood cells. When the oscillation frequency is sufficiently large (Fig. 5b, c, e and f), the catheter radius $\hat{d}=0.3$ could be viewed as a critical level, below which the steady shear stress distribution is only mildly affected by the catheter size, but when it is exceeded, the shear stress will tend to increase in magnitude dramatically with the catheter size. Similar trends can also be found for the steady pressure gradient as has been illustrated in Fig. 4. As a matter of fact, a catheter of radius $\hat{d}=0.3$ will take up only $9 \%$ of the cross-sectional area of the tube. An order-of-magnitude analysis will show that, with a blockage ratio equal to $10 \%$, the increase in the section-mean velocity is also of the order $10 \%$. Such a limited increase in velocity will also lead to a limited increase in the magnitude of the dynamics quantities. The results here suggest that in practice a catheter radius no greater than $\hat{d}=0.3$ should be used in order to avoid too large an increase in pressure or stress to be induced in the fluid.

\section{Concluding remarks}

In this paper, we have examined the oscillatory as well as the steady Lagrangian flows of a fluid as induced by a wave propagating in the annulus of a catheterized viscoelastic tube. The problem is entirely Lagrangian in formulation and analysis. The steady axial velocity has been solved as an analytical function of the wall and fluid properties, the Womersley number, and the catheter radius, as given in Eq. (71) in the second-order problem. Because of wave damping, all the time-mean quantities contain an exponential factor for axial decay along the tube. The influences of the oscillation frequency and the catheter radius on steady streaming velocity, steady pressure gradient and steady shear stress have been studied. A catheter radius that is $30 \%$ of the radius of the inner wall surface is a possibly critical radius, below which the steady pressure gradient and the steady shear stress in most part of the fluid may not be appreciably affected by the presence of the catheter. 
Extension of the present model is possible on taking into account additional effects due to non-Newtonian fluid, large amplitude wave, stenosis and curvature of the artery wall. For a problem with complex geometry or strong nonlinearity, numerical efforts should be employed. Our analytical results presented here can be used as a benchmark to test the accuracy of a computational scheme for solving the Lagrangian problem numerically.

\section{Acknowledgments}

The work was supported by the Research Grants Council of the Hong Kong Special Administrative Region, China, through Project No. HKU 715609E. This study constitutes part of the work performed by the first author for his Ph.D. thesis under the supervision of the second author at the University of Hong Kong. 


\section{References}

[1] SARKAR A. and JAYARAMAN G. Correction to flow rate - pressure drop relation in coronary angioplasty: steady streaming effect[J]. J. Biomech., 1998, 31: $781-791$.

[2] SARKAR A. and JAYARAMAN G. Nonlinear analysis of oscillatory flow in the annulus of an elastic tube: Application to catheterized artery[J]. Phys. Fluids, 2001, 13(10): 2901-2911.

[3] SHARP M. K. and MOHAMMAD S. F. Scaling of hemolysis in needles and catheters[J]. Ann. Biomed. Eng., 1998, 26(5): 788-797.

[4] JAYARAMAN G. and SARKAR A. Nonlinear analysis of arterial blood flow steady streaming effect[J]. Nonlinear Anal., 2005, 63: 880-890.

[5] JAYARAMAN G. and DASH R. K. Numerical study of flow in a constricted curved annulus: An application to flow in a catheterised artery $[\mathrm{J}]$. J. Eng. Math., 2001, 40: 355-376.

[6] SANKAR D. S. A two-fluid model for pulsatile flow in catheterized blood vessels[J]. Int. J. Non-linear Mech., 2009, 44: 337-351.

[7] RILEY N. Steady streaming[J]. Annu. Rev. Fluid Mech., 2001, 33: 43-65.

[8] SARKAR A. and JAYARAMAN G. The effect of wall absorption on dispersion in oscillatory flow in an annulus: application to a catheterized artery[J]. Acta Mech., 2004, 172: 151-167.

[9] DRAGON C. A. and GROTBERG J. B. Oscillatory flow and mass transport in a flexible tube[J]. J. Fluid Mech., 1991, 231: 135-155.

[10] MA Y. and NG C.O. Wave propagation and induced steady streaming in viscous fluid contained in a prestressed viscoelastic tube[J]. Phys. Fluids, 2009, 21(5): 051901.

[11] NG C.O. and ZHANG X. Mass transport in water waves over a thin layer of soft viscoelastic mud[J]. J. Fluid Mech., 2007, 573: 105-130. 
[12] ZHANG X. and NG C. O. On the oscillatory and mean motions due to waves in a thin viscoelastic layer[J]. Wave Motion, 2006, 43: 387-405.

[13] BATRA R. C. and YU J. H. Effect of inertia forces on the damping of a constrained layer finitely deformed in shearing[J]. J. Sound Vib., 2001, 241: 913919.

[14] MACOSKO C. W. Rheology: Principles, Measurements and Applications[M]. New York: Wiley, 1994.

[15] DEMIRAY H. A viscoelastic model for arterial wall materials[J]. Int. J. Eng. Sci., 1994, 32: 1567-1578.

[16] GUNDIAH N., RATCLIfFE M. B. and PRUITT L. A. The biomechanics of arterial elastin[J]. J. Mech. Behav., 2009, 2: 288-296.

[17] PIERSON W. J. Perturbation analysis of the Navier-Stokes equations in Lagrangian form with selected linear solution $[J]$. J. Geophys. Res., 1962, 67: $3151-3160$.

[18] LING S. C. and ATABEK H. B. A nonlinear analysis of pulsatile flow in arteries[J]. J. Fluid Mech., 1972, 55: 493-511.

[19] COX R. H. Wave propagation through a Newtonian fluid contained within thick-walled, viscoelastic tube[J]. Biophys. J., 1968, 8: 691-709.

[20] WILMER W. N. and MICHAEL F. O. McDonald's Blood Flow in Arteries: Theoretical, Experimental, and Clinical Principles[M]. London: Arnold, 1998. 


\section{Figure captions}

Figure 1 Schematic diagram of the problem, where description is based on Lagrangian axial and radial coordinates, $Z$ and $R$. Viscous fluid contained in the annulus between a viscoelastic tube and a rigid catheter is subjected to time-periodic oscillatory pressure forcing at $Z=0$ : (a) longitudinal-section view; (b) cross-section view. A fluid element initially centered at $(R, Z)$, on undergoing deformation, moves to a new center $(r, z)$ at time $t$.

Figure 2 Phase velocity $C_{1}(\mathrm{~m} / \mathrm{s})$ and imaginary wavenumber $\hat{k}_{i}$ of the Young wave as functions of the Womersley number $\alpha$ for the free-tube case in (a) and (b), and for the tethered-tube case in (c) and (d), where $\hat{d}$ varies from 0 to 0.5 .

Figure 3 Cross-sectional profiles of steady axial velocity at $\hat{Z}=0$, for catheter radius $\hat{d}=0$ (solid), 0.1 (dashes), 0.3 (dash-dots), 0.5 (dash-dot-dots), and the Womersley number $\alpha=2,6,10$, when the tube is free of stress, as in (a), (b), (c), respectively, or when the tube is tethered, as in (d), (e), (f), respectively.

Figure 4 Cross-sectional profiles of steady pressure gradient at $\hat{Z}=0$, for catheter radius $\hat{d}=0$ (solid), 0.1 (dashes), 0.3 (dash-dots), 0.5 (dash-dot-dots), and the Womersley number $\alpha=2,6,10$, in (a), (b), (c), respectively.

Figure 5 Cross-sectional profiles of steady shear stress $\tau_{f}^{s}(\hat{R})=\overline{\hat{\tau}}_{f r z 2}(\hat{R}, \hat{Z}=0)$, for catheter radius $\hat{d}=0$ (solid), 0.1 (dashes), 0.3 (dash-dots), 0.5 (dash-dot-dots), and the Womersley number $\alpha=2,6,10$ in (a), (b), (c), respectively. Steady shear stress at the inner wall surface $\tau_{w s s}=\tau_{f}^{s}(\hat{R}=1)$ (solid), and steady shear stress at the catheter surface $\tau_{c s s}=\tau_{f}^{s}(\hat{R}=\hat{d})$ (dashes) as functions of the catheter radius $\hat{d}$, for the Womersley number $\alpha=2,6,10$ in (d), (e), (f), respectively. 
(a)

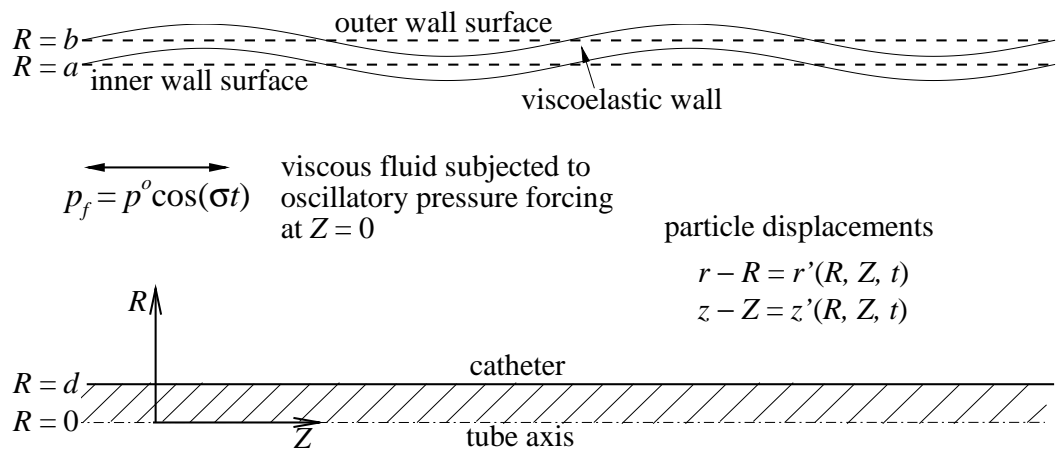

(b)

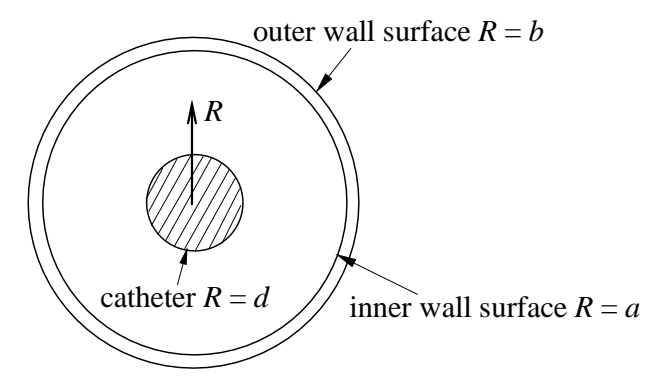

Figure 1: Schematic diagram of the problem, where description is based on Lagrangian axial and radial coordinates, $Z$ and $R$. Viscous fluid contained in the annulus between a viscoelastic tube and a rigid catheter is subjected to time-periodic oscillatory pressure forcing at $Z=0$ : (a) longitudinal-section view; (b) cross-section view. A fluid element initially centered at $(R, Z)$, on undergoing deformation, moves to a new center $(r, z)$ at time $t$. 

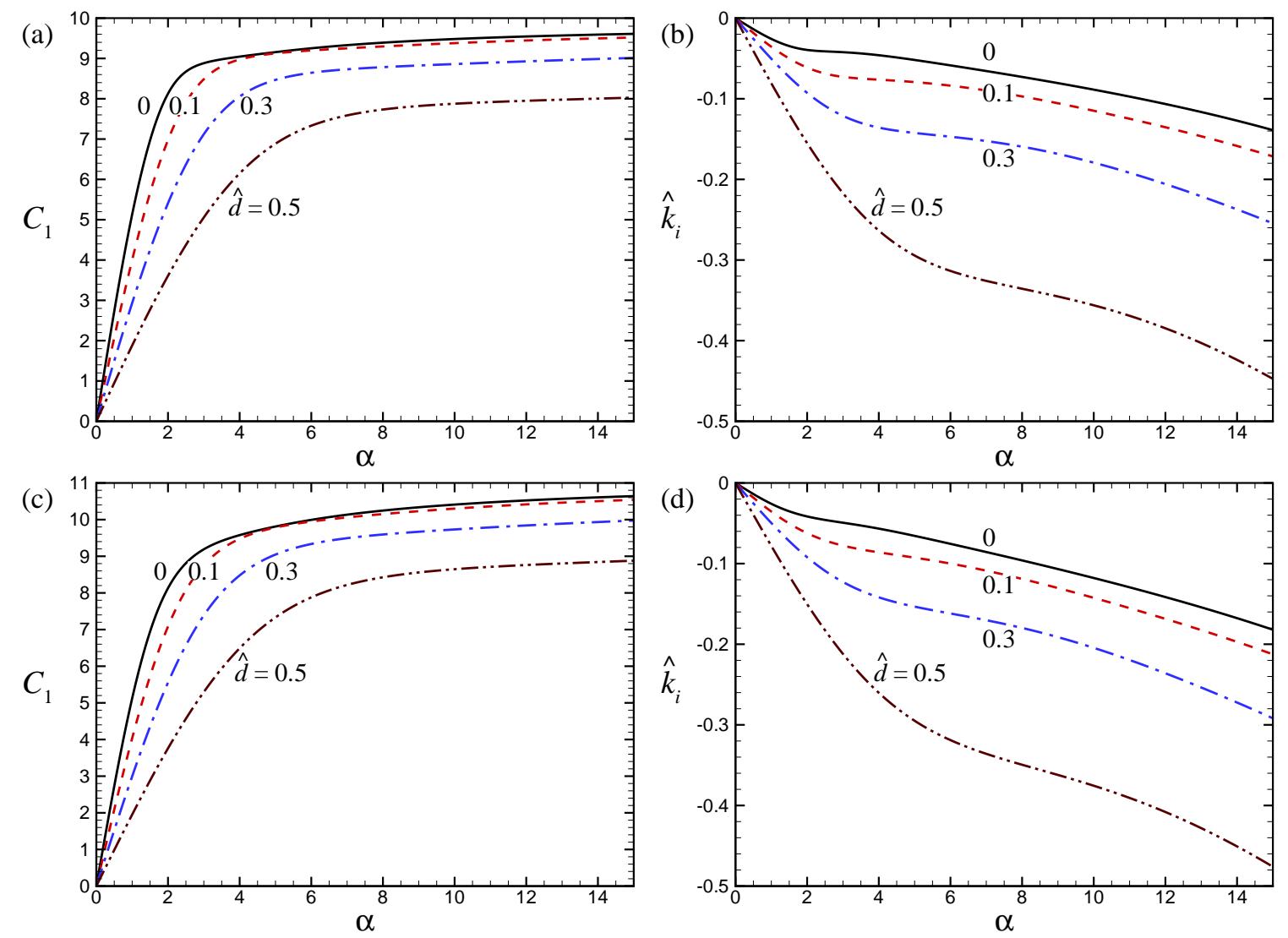

Figure 2: Phase velocity $C_{1}(\mathrm{~m} / \mathrm{s})$ and imaginary wavenumber $\hat{k}_{i}$ of the Young wave as functions of the Womersley number $\alpha$ for the free-tube case in (a) and (b), and for the tethered-tube case in (c) and (d), where $\hat{d}$ varies from 0 to 0.5 . 

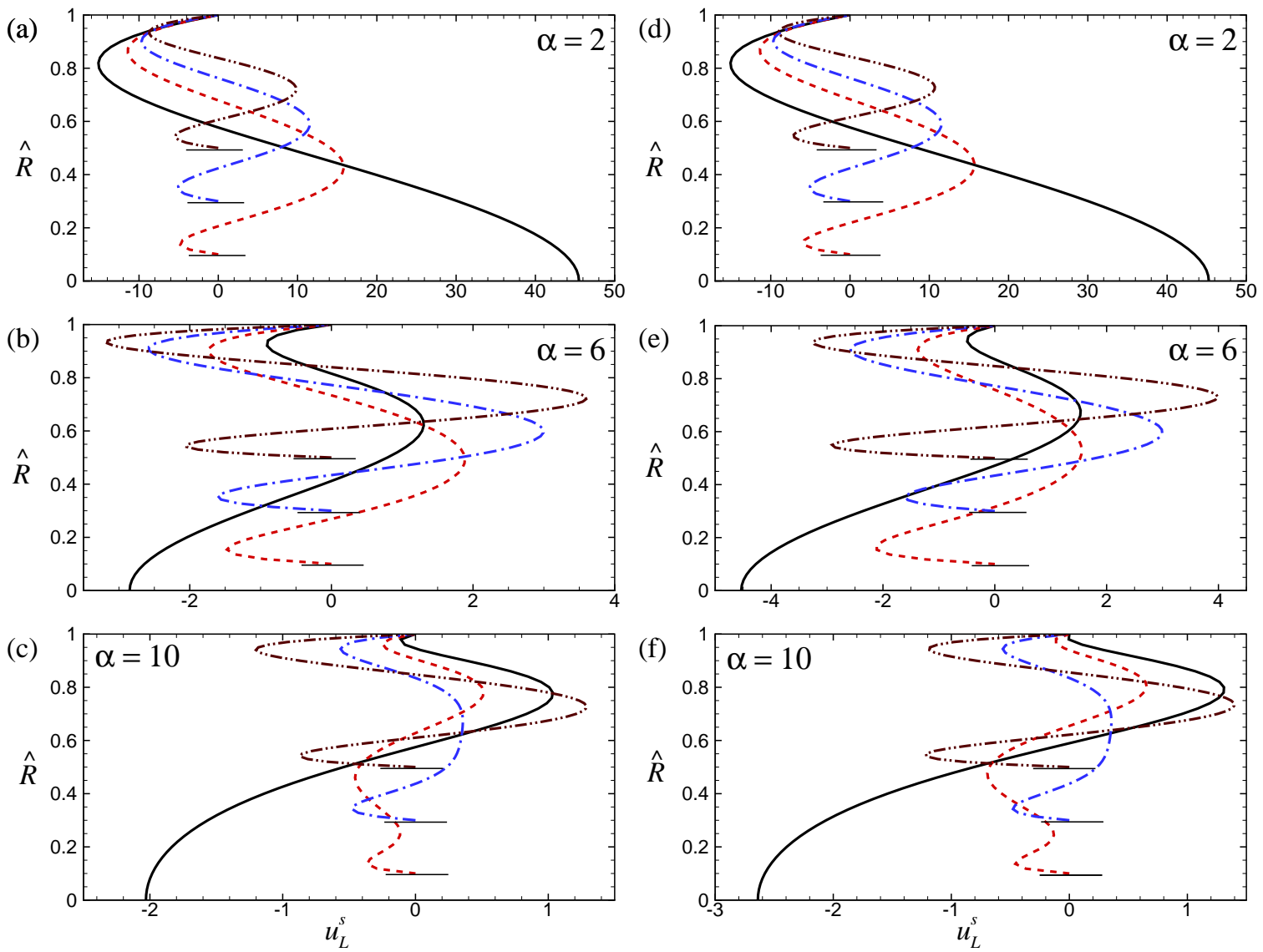

Figure 3: Cross-sectional profiles of steady axial velocity at $\hat{Z}=0$, for catheter radius $\hat{d}=0$ (solid), 0.1 (dashes), 0.3 (dash-dots), 0.5 (dash-dot-dots), and the Womersley number $\alpha=2,6,10$, when the tube is free of stress, as in (a), (b), (c), respectively, or when the tube is tethered, as in (d), (e), (f), respectively. 

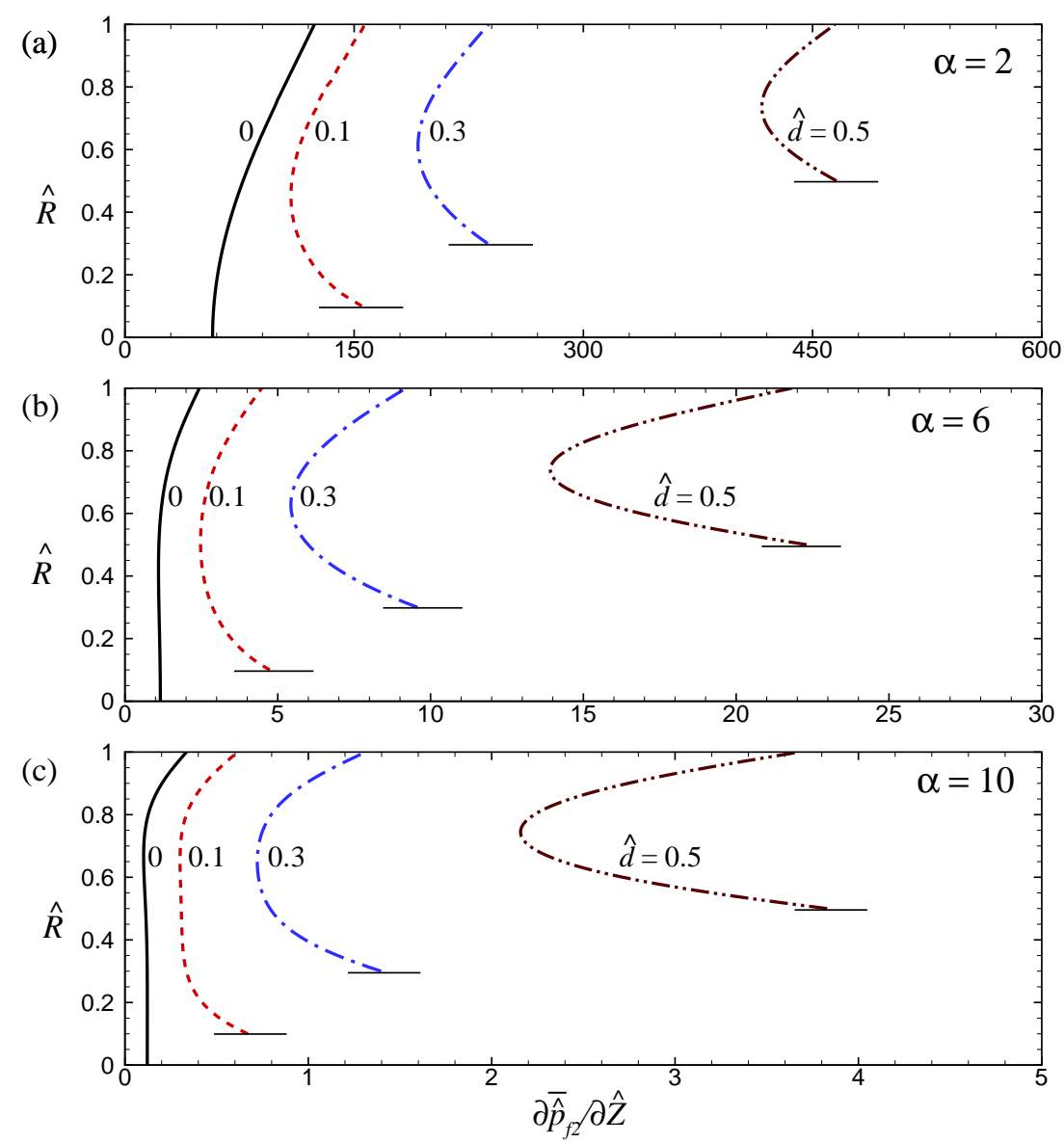

Figure 4: Cross-sectional profiles of steady pressure gradient at $\hat{Z}=0$, for catheter radius $\hat{d}=0$ (solid), 0.1 (dashes), 0.3 (dash-dots), 0.5 (dash-dot-dots), and the Womersley number $\alpha=2,6,10$, in (a), (b), (c), respectively. 

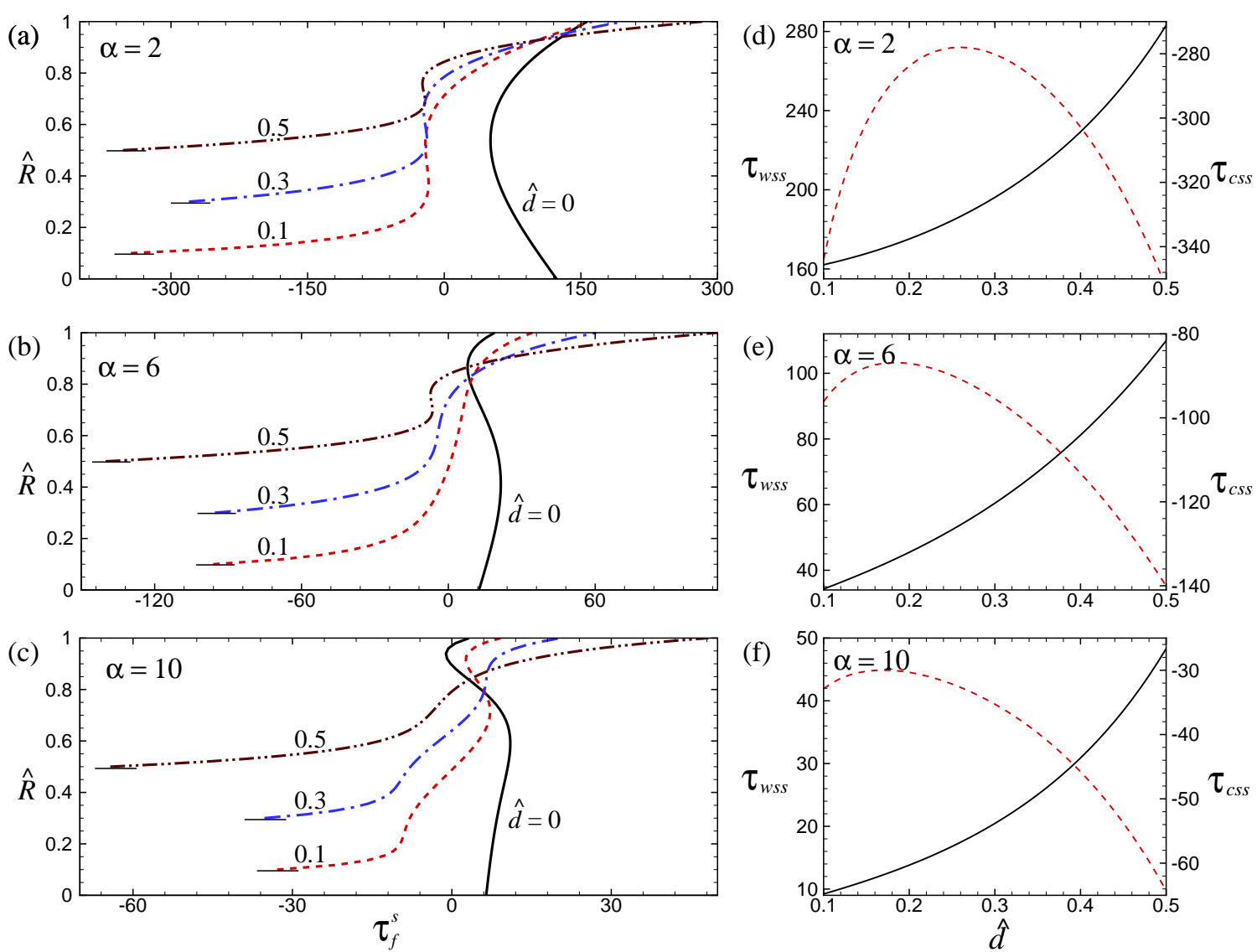

Figure 5: Cross-sectional profiles of steady shear stress $\tau_{f}^{s}(\hat{R})=\overline{\hat{\tau}}_{f r z 2}(\hat{R}, \hat{Z}=0)$, for catheter radius $\hat{d}=0$ (solid), 0.1 (dashes), 0.3 (dash-dots), 0.5 (dash-dot-dots), and the Womersley number $\alpha=2,6,10$ in (a), (b), (c), respectively. Steady shear stress at the inner wall surface $\tau_{w s s}=\tau_{f}^{s}(\hat{R}=1)$ (solid), and steady shear stress at the catheter surface $\tau_{c s s}=\tau_{f}^{s}(\hat{R}=\hat{d})$ (dashes) as functions of the catheter radius $\hat{d}$, for the Womersley number $\alpha=2,6,10$ in (d), (e), (f), respectively. 